\title{
SPACES DOMINATED BY TWO-COMPLEXES
}

\author{
SUSHIL JAJODIA
}

\begin{abstract}
We say a group $G$ is of geometric dimension $<2$ if there is an aspherical 2-dimensional $C W$-complex $P$ with fundamental group isomorphic to $G$. In this note, we study the following problem: Suppose $G$ is a group of geometric dimension $<2$ with associated aspherical 2-dimensional $C W$-complex $P$. Suppose further that $X$ is a $\mathbf{C W}$-complex having fundamental group isomorphic to $G$ and that $X$ is dominated by a 2-complex. If the Wall invariant $\mathrm{Wa}_{2}[X] \in \tilde{K}_{0}(Z G)$ vanishes, does $X$ have the same homotopy type as $P \vee k S^{2}$ where $k S^{2}$ denotes the sum of $k$ copies of the 2-sphere $S^{2}$ ?
\end{abstract}

Throughout this paper, all $\mathrm{CW}$-complexes are finite connected with some zero cell chosen as base point, all maps preserve base points, and all homotopies are relative to base points.

We say a group $G$ is of geometric dimension $<2$ if there is an aspherical 2-dimensional $\mathrm{CW}$-complex $\boldsymbol{P}$ with fundamental group isomorphic to $\boldsymbol{G}$. In this note, we study the following problem: Suppose $G$ is a group of geometric dimension $\leqslant 2$ with associated aspherical 2-dimensional $C W$-complex $P$. Suppose further that $X$ is a $\mathrm{CW}$-complex having fundamental group isomorphic to $G$ and that $X$ is dominated by a 2-complex. If the Wall invariant $\mathrm{Wa}_{2}[X] \in \tilde{K}_{0}(Z G)$ vanishes (see [7]), does $X$ have the same homotopy type as $P \vee k S^{2}$ where $k S^{2}$ denotes the sum of $k$ copies of the 2-sphere $S^{2}$ ?

The answer to this question, in general, is no. In fact, Dunwoody [1] has constructed a 2-complex $X$ which is not homotopy equivalent to $P \vee S^{2}$ where $P$ denotes the geometric realization of the presentation $\left(a, b: a^{2} b^{-3}\right)$ for the trefoil group.

Lemma. Let $G, P$ and $X$ be as above. Then $\pi_{2} X$ as a $Z G$-module is stably free, i.e., $\pi_{2} X \oplus Z G^{k} \simeq Z G^{l}$ for some $k$ and $l$.

Proof. Because the Wall invariant of $X$ is zero, we may assume that $X$ is a finite 3-complex [7, Theorem F, p. 66]. By [2, Theorem 4.1, p. 236] or [8, Theorem 1, p. 409], for some $m, X \vee m S^{2}$ is homotopy equivalent to $Z$ where $Z$ is a 2-complex with $\pi_{1} Z \simeq G$. Since $Z$ and $P$ are both 2-complexes with isomorphic fundamental groups, by a well-known result of J. H. C. Whitehead, $Z \vee t S^{2} \simeq P \vee l S^{2}$ for some $t$ and $l$. Therefore, $X \vee(m+t) S^{2} \simeq Z \vee t S^{2} \simeq P \vee l S^{2}$ so that $\pi_{2} X \oplus$ $Z G^{k} \simeq \pi_{2} P \oplus Z G^{l}$ (where $k=m+t$ ). But $P$ is aspherical, therefore $\pi_{2} P=0$, hence $\pi_{2} X \oplus Z G^{k} \simeq Z G^{l}$.

Received by the editors February 22, 1980 and, in revised form, June 11, 1980.

AMS (MOS) subject classifications (1970). Primary 55A20. 
THEOREM 1. Let $X$ and $P$ be as above. If Euler characters $\chi(X)=\chi(P)$, then $X$ and $P$ have the same homotopy type.

Proof. Since $\chi(X)=\chi(P)$, we must have $k=l$ and $\pi_{2} X \oplus Z G^{k}=Z G^{k}$. By a result of Kaplansky [6], this means that $\pi_{2} X=0$. Because $X$ is dominated by a 2-complex $Y$, the universal cover $\tilde{X}$ is dominated by a 2-complex $\tilde{Y}$ (this follows from the unique homotopy lifting property and the fact that we are working in the based category). Thus we have the commutative diagram

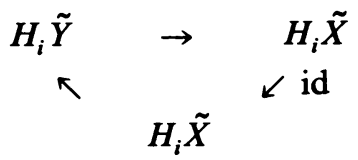

This means that the map $H_{i} \tilde{Y} \rightarrow H_{i} \tilde{X}$ is onto. Since $H_{i} \tilde{Y}=0$ for $i>3, H_{i} \tilde{X}=0$ for $i \geqslant 3$. Therefore $\tilde{X} \simeq *$, and so $X$ is aspherical. The result now follows.

THEOREM 2. Let $X$ be a CW-complex with fundamental group free abelian of rank two. If $X$ is dominated by a 2-complex, then $X$ is homotopy equivalent to $P \vee t S^{2}$ where $P$ is the torus (i.e., geometric realization of the presentation $\left(a, b: a b a^{-1} b^{-1}\right)$ of $Z \times Z)$.

PROoF. For any finitely generated free abelian group $H$, all finitely generated projective $Z H$-modules are free. Therefore, $\tilde{K}_{0}(Z \times Z)=0$ and, by the Lemma above, $\pi_{2} X \simeq Z G^{l-k}$. Now it is possible to construct a homotopy equivalence between $X$ and $P \vee(l-k) S^{2}$ using [5, Theorem 3, p. 26].

EXAMPLE. Let $X$ denote the complement $S^{4}-k\left(S^{2}\right)$ of the spun trefoil knot $\left(S^{4}, k\left(S^{2}\right)\right)$ where $k\left(S^{2}\right) \subset S^{4}$ is a 2 -sphere formed by spinning the trefoil knot about the standard 2-sphere $S^{2}$. Then we can obtain a 3-complex $K$ which is a deformation retract of $X$ and such that the Euler character $\chi(K)=0$ (see [4] for details). Because $\pi_{2} X \neq 0$, in view of Theorem $1, K$ cannot be dominated by a 2-complex.

In the Lemma above, we saw that $\pi_{2} X \oplus Z G^{k} \simeq Z G^{l}$. This implies that $X \vee$ $k S^{2} \simeq P \vee l S^{2}$. In order that $X \simeq P \vee(l-k) S^{2}$, we must have that $\pi_{2} X$ is actually free. Unfortunately, $\pi_{2} X$ need not be free. The 2-complex $X$ in Dunwoody's example (see above) is such that $\pi_{2} X \oplus Z G \simeq Z G^{2}$, yet $\pi_{2} X$ is not isomorphic to $Z G$. We give below a criterion which insures the freeness of this module in the case when $l \geqslant 2 k$.

TheOrem 3. Let $G, P$, and $X$ be as above with $l \geqslant 2 k$. Then $X \simeq P \vee(l-k) S^{2}$ if and only if there is a homotopy equivalence $f: X \vee k S^{2} \rightarrow P \vee l S^{2}$, which on the second homotopy group induces the projection map, i.e., the following diagram

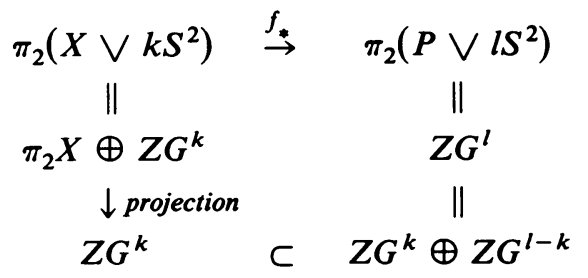

commutes such that there is a $Z G$-basis $\left\{d_{1}, \ldots, d_{l}\right\}$ of $\pi_{2} X \oplus Z G^{k}\left(\simeq Z G^{l}\right)$ such that for some $s \leqslant l-k$, the images $\left\{f_{*} d_{1}, \ldots, f_{*} d_{s}\right\}$ generate $Z G^{k} \subset Z G^{l}$. 
Proof. If $X \simeq P \vee(l-k) S^{2}$, then $\pi_{2} X$ is isomorphic to $Z G^{l-k}$. Hence it is possible to construct a desired homotopy equivalence $f$ using Theorem 3, p. 236 of [5]. Conversely, suppose there is a homotopy equivalence $f: X \vee k S^{2} \rightarrow P \vee l S^{2}$ having the property stated in the theorem. By Gabel's Lemma [3, p. 39], it follows that $\pi_{2} X \simeq Z G^{l-k}$. Hence $X \simeq P \vee(l-k) S^{2}$.

I wish to thank Professor M. N. Dyer for his suggestions for the improvement of this paper.

\section{REFERENCES}

1. M. J. Dunwoody, The homotopy type of a two-dimensional complex, Bull. London Math. Soc. 8 (1976), 282-285.

2. M. N. Dyer, On the 2-realizability of 2-types, Trans. Amer. Math. Soc. 204 (1975), 229-243.

3. T. Y. Lam, Series summation of stably free modules, Quart. J. Math. Oxford Ser. (2) 27 (1976), 37-46.

4. S. J. Lomonaco, Jr., The second homotopy group of a spun knot, Topology 8 (1969), 95-98.

5. S. Mac Lane and J. H. C. Whitehead, On the 3-type of a complex, Proc. Nat. Acad. Sci. U.S.A. 36 (1950), 41-48.

6. M. S. Montgomery, Left and right inverses in group algebras, Bull. Amer. Math. Soc. 75 (1969), 539-540.

7. C. T. C. Wall, Finiteness conditions for CW-complexes, Ann. of Math. (2) 81 (1965), 56-69.

8. J. M. Cohen, Complexes dominated by a 2-complex, Topology 16 (1977), 409-415.

Department of Mathematics, University of Wisconsin-Stevens Point, Stevens Point, WisconSIN 54481 